\title{
Predestined Dark Matter in Gauge Extensions of the Standard Model
}

\author{
Ernest Ma \\ Physics and Astronomy Department, University of California, Riverside, California 92521, USA \\ Jockey Club Institute for Advanced Study, Hong Kong University of Science and Technology, Hong Kong, China
}

Received: 15 March 2018, Accepted: 2 April 2018, Published: 12 May 2018

\begin{abstract}
In any gauge extension of the standard model (SM) of quarks and leptons, there is a minimal set of fermion and scalar multiplets which encompasses all the particles and interactions of the SM. Included within this set, there may be a suitable dark-matter candidate. If not, one may still exist from the judicious addition of a simple fermion or scalar multiplet without any imposed symmetry. Some new examples of such predestined dark matter are discussed.
\end{abstract}

\section{DOI: 10.31526/LHEP.1.2018.01}

Automatic (or predestined) symmetries play an important part in the structure of particle physics. In the context of the standard $S U(3)_{C} \times S U(2)_{L} \times U(1)_{Y}$ gauge model of quarks and leptons (SM), baryon number $B$ and lepton number $L$ are automatically conserved because of the chosen particle content under the gauge symmetry. Furthermore, $C P$ conservation in the hadronic sector would also be automatic if there were only two families of quarks. If the seesaw mechanism is invoked to obtain very small Majorana neutrino masses by increasing the particle content with the addition of heavy right-handed Majorana singlet neutrinos, then lepton number $L$ is replaced with lepton parity $(-1)^{L}$. Suppose the particle content is increased again with a fermion or scalar multiplet, what new phenomena would occur?

It was shown already some years ago [1] that the neutral member of a fermion quintet or a scalar septet would be a good dark-matter candidate. Either addition to the SM without any imposed symmetry would imply an automatic dark parity which keeps this neutral component (which is also automatically the lightest) against possible decay. Whereas this is an attractive idea phenomenologically, the requirement of such large fundamnetal multiplets is not quite so attractive theoretically.

In this paper, the notion of predestined dark matter is explored in the context of some left-right extensions of the SM, as well as gauge $U(1)$ extensions with heavy Majorana singlet or triplet fermions as the seesaw anchors of small neutrino masses. It will be shown that simple solutions exist in both cases. Some are already known and others are new.

Consider the well-known conventional left-right extension of the SM. Under $S U(3)_{C} \times S U(2)_{L} \times S U(2)_{R} \times U(1)$,

$$
\begin{aligned}
& \left(\begin{array}{l}
u \\
d
\end{array}\right)_{L} \sim\left(3,2,1, \frac{1}{6}\right), \quad\left(\begin{array}{l}
u \\
d
\end{array}\right)_{R} \sim\left(3,1,2, \frac{1}{6}\right), \\
& \left(\begin{array}{l}
v \\
e
\end{array}\right)_{L} \sim\left(1,2,1,-\frac{1}{2}\right), \quad\left(\begin{array}{l}
v \\
e
\end{array}\right)_{R} \sim\left(1,1,2,-\frac{1}{2}\right) .
\end{aligned}
$$

The only fermion additions to the SM are the right-handed neutrinos $v_{R}$, and the $U(1)$ gauge symmetry is seen to be just

\footnotetext{
Email address: ma@phyun8.ucr.edu (Ernest Ma)

${ }^{1}$ (C) 2018 The Author(s). Published by LHEP. This is an open access article under the CC BY licensehttp://creativecommons .org/licenses/by/4.0/
}

$(B-L) / 2$. To obtain fermion masses, at least one scalar bidoublet

$$
\eta=\left(\begin{array}{ll}
\eta_{1}^{0} & \eta_{2}^{+} \\
\eta_{1}^{-} & \eta_{2}^{0}
\end{array}\right) \sim(1,2,2,0)
$$

is needed. The choice of the rest of the scalar sector depends on how neutrinos acquire mass. All possible such scenarios have been discussed some years ago [2].

Suppose an $S U(2)_{R}$ scalar doublet $\Phi_{R}=\left(\phi_{R}^{+}, \phi_{R}^{0}\right)$ is added [3], then the particle content of this model guarantees also $B$ and $L$ conservation, and neutrinos are Dirac particles. Suppose instead an $S U(2)_{R}$ scalar triplet $\xi_{R}=\left(\xi_{R}^{++}, \xi_{R}^{+}, \xi_{R}^{0}\right)$ is used, then $v_{R}$ acquires a large Majorana mass and $L$ is broken to $(-1)^{L}$, resulting in the canonical seesaw mechanism for small Majorana neutrino masses.

Consider now the addition of a fermion bidoublet

$$
\psi=\left(\begin{array}{ll}
\psi_{1}^{0} & \psi_{2}^{+} \\
\psi_{1}^{-} & \psi_{2}^{0}
\end{array}\right) \sim(1,2,2,0)
$$

without any imposed symmetry. It may be assumed either lefthanded or right-handed, because it is self-dual. It may then connect to $(\nu, e)_{L}$ through $\Phi_{R}$, in which case no new automatic symmetry arises. However, if $\xi_{R}$ is used instead, there is no such connection. Hence, the fermion bidoublet $\psi$ has its own conserved $U(1)$ symmetry, with $\psi_{1}^{0} \psi_{2}^{0}-\psi_{1}^{-} \psi_{2}^{+}$as an invariant mass term. Again it can be shown [4] that the neutral member $\psi_{1,2}^{0}$ is lighter than the charged member, so that the former is a simple predestined dark-matter candidate. Now it appears that $\psi_{1,2}^{0}$ is a Dirac fermion and couples to the SM $Z$ boson. It would then be ruled out by direct-detection experiments by many orders of magnitude. However, as already pointed out [5], this $U(1)$ is broken radiatively to $Z_{2}$ from $W_{L}-W_{R}$ mixing. Hence $\psi_{1,2}^{0}$ splits up to two Majorana fermions and the direct-detection limit from the $Z$ boson does not apply.

Consider next a fermion triplet under $S U(2)_{L}$ or $S U(2)_{R}$ :

$$
\begin{aligned}
& \Sigma_{L}=\left(\Sigma_{L}^{+}, \Sigma_{L}^{0}, \Sigma_{L}^{-}\right) \sim(1,3,1,0), \\
& \Sigma_{R}=\left(\Sigma_{R}^{+}, \Sigma_{R}^{0}, \Sigma_{R}^{-}\right) \sim(1,1,3,0) .
\end{aligned}
$$

If $\Phi_{R}$ is available, then $\Sigma_{R}$ may be connected to $(\nu, e)_{R}$ and would not generate any new symmetry. However, the absence [3] of an $S U(2)_{L}$ scalar doublet $\Phi_{L}$ means that $\Sigma_{L}$ would have a conserved symmetry, i.e. $Z_{2}$ because $\Sigma_{L}$ is a Majorana triplet without any imposed symmetry. Note that $\Sigma_{L}^{0}$ does not 
couple to the SM $Z$ or the scalar bidoublet $\eta$. Its only interaction is with $\Sigma_{L}^{ \pm}$through the $\mathrm{SM} W_{L}^{ \pm}$bosons. This would allow it to become a viable dark-matter candidate as shown some years ago [6]. The important difference is that $Z_{2}$ was imposed in that model of scotogenic neutrino mass, whereas here it is predestined.

For the choice of an scalar triplet $\xi_{R}$ in breaking $S U(2)_{R}$, $\Sigma_{R}$ does not connect to $(v, e)_{R}$. Hence $\Sigma_{R}^{0}$ is now also a viable dark-matter candidate. Its presence has been discussed [7, 8], which may also be motivated by $S O(10)$ unification. For completeness, a fermion singlet

$$
S \sim(1,1,1,0)
$$

would also have an automatic discrete $Z_{2}$ symmetry in this case. However, $S$ has no interaction by itself and would not have the correct dark-matter relic abundance from thermal freezeout.

As for possible scalar dark matter, consider again a triplet under $S U(2)_{L}$ or $S U(2)_{R}$ :

$$
\begin{aligned}
& \chi_{L}=\left(\chi_{L}^{+}, \chi_{L}^{0}, \chi_{L}^{-}\right) \sim(1,3,1,0), \\
& \chi_{R}=\left(\chi_{R}^{+}, \chi_{R}^{0}, \chi_{R}^{-}\right) \sim(1,1,3,0) .
\end{aligned}
$$

If $\Phi_{R}$ or $\xi_{R}$ is available, then $\chi_{R}$ couples to the triplet decomposition of $\Phi_{R}^{\dagger} \Phi_{R}$ or $\xi_{R}^{\dagger} \xi_{R}$ and would not generate any new symmetry. Conversely, if $\Phi_{L}$ or $\xi_{L}$ is absent, $\chi_{L}$ would have a conserved $Z_{2}$ symmetry, and the real scalar $\chi_{L}^{0}$ would be predestined dark matter.

The results of this section are summarized in Table 1. Of the

\begin{tabular}{|c|c|c|c|c|c|}
\hline$\eta$ & $\Phi_{R}$ & $\Phi_{L}$ & $\xi_{R}$ & $\xi_{L}$ & PDM \\
\hline$\sqrt{ }$ & $\sqrt{ }$ & $\sqrt{ }$ & - & - & - \\
$\sqrt{ }$ & $\sqrt{ }$ & - & - & - & $\Sigma_{L,} \chi_{L}$ \\
\hline$\sqrt{ }$ & - & - & $\sqrt{ }$ & $\sqrt{ }$ & $S, \psi, \Sigma_{L, R}$ \\
$\sqrt{ }$ & - & - & $\sqrt{ }$ & - & $S, \psi, \Sigma_{L, R}, \chi_{L}$ \\
\hline
\end{tabular}

TABLE 1: Predestined dark matter (PDM) from left-right scalar content.

possible dark-matter candidates, the neutral fermion or scalar member of an $S U(2)_{L}$ triplet is the most viable because it interacts with its charged member through the SM $W_{L}^{ \pm}$boson. Higher multiplets are of course also possible. For example, an $S U(2)_{R}$ fermion quintet has recently been considered [9]. The left-right model may be embedded in $S O(10)$ in which case there is no need for new fermions. It may also be embedded in $E_{6}$ using its fundamental 27 representation which decomposes into $\left(3,3^{*}, 1\right)+\left(1,3,3^{*}\right)+\left(3^{*}, 1,3\right)$ under its maximal subgroup $S U(3)_{C} \times S U(3)_{L} \times S U(3)_{R}$ :

$$
\left(\begin{array}{lll}
d & u & h \\
d & u & h \\
d & u & h
\end{array}\right),\left(\begin{array}{ccc}
\psi_{1}^{0} & \psi_{2}^{+} & v \\
\psi_{1}^{-} & \psi_{2}^{0} & e \\
v^{c} & e^{c} & s
\end{array}\right),\left(\begin{array}{lll}
d^{c} & d^{c} & d^{c} \\
u^{c} & u^{c} & u^{c} \\
h^{c} & h^{c} & h^{c}
\end{array}\right)
$$

Hence the particle content has automatically new fermions. In terms of $S O(10) \times U(1)_{\psi}$, the 27 decomposes to $(16,1)+$ $(10,-2)+(1,4)$. The further decomposition to $S U(5) \times U(1)_{\chi}$ is then $16=\left(5^{*}, 3\right)+(10,-1)+(1,-5), 10=\left(5^{*},-2\right)+(5,2)$ and $1=(1,0)$. Consider the assignments of the fundamental fermions of Eq. (10) under $U(1)_{\psi}$ and $U(1)_{\chi}$ :

$$
\begin{aligned}
U(1)_{\psi}: & \left(\begin{array}{ccc}
1 & 1 & -2 \\
1 & 1 & -2 \\
1 & 1 & -2
\end{array}\right),\left(\begin{array}{ccc}
-2 & -2 & 1 \\
-2 & -2 & 1 \\
1 & 1 & 4
\end{array}\right), \\
& \left(\begin{array}{ccc}
1 & 1 & 1 \\
1 & 1 & 1 \\
-2 & -2 & -2
\end{array}\right), \\
U(1)_{\chi}: & \left(\begin{array}{ccc}
-1 & -1 & 2 \\
-1 & -1 & 2 \\
-1 & -1 & 2
\end{array}\right),\left(\begin{array}{ccc}
-2 & 2 & 3 \\
-2 & 2 & 3 \\
-5 & -1 & 0
\end{array}\right), \\
& \left(\begin{array}{ccc}
3 & 3 & 3 \\
-1 & -1 & -1 \\
-2 & -2 & -2
\end{array}\right) .
\end{aligned}
$$

This shows that the known quarks and leptons are odd while the other fermions are even under $U(1)_{\psi}$. Calling this charge $D_{\psi}$, the derived parity [10]

$$
R_{\psi}=(-1)^{D_{\psi}+2 j}
$$

where $j=1 / 2$ is the spin of the particle, may then be used as the stabilizing dark parity of this model. Note that the singlet fermion $S$ and the bidoublet fermion $\psi$ discussed earlier are already part of the $\left(1,3,3^{*}\right)$ multiplet. Hence this is an example of possible predestined dark matter within a given gauge symmetry and its particle content. However, it has been pointed out recently [11] that in the course of breaking $[S U(3)]^{3}$ to the SM using only bifundamental scalars, $R_{\psi}$ is only preserved with an imposed $Z_{2}$ symmetry. This invalidates $S$ or $\psi$ as predestined dark matter. Another closely related example is also known [12].

Under $U(1)_{\chi}$, there appears to be a possible residual $U(1)$ symmetry, under which $h, \psi_{2}^{+}, \psi_{2}^{0}$ and $h^{c}, \psi_{1}^{0}, \psi_{1}^{-}$transform oppositely, whereas others are trivial. Again the bifundamental scalars used in the symmetry breaking would spoil this scenario without an imposed symmetry.

Because there are two copies of $5^{*}$ of $S U(5)$ in $E_{6}$, an alternative left-right model (ALRM) [13] is possible. At the $S U(2)_{L} \times S U(2)_{R} \times U(1)_{X}$ level, the quarks and leptons are then given by

$$
\begin{aligned}
& \left(\begin{array}{l}
u \\
d
\end{array}\right)_{L} \sim\left(2,1, \frac{1}{6}\right),\left(\begin{array}{l}
u \\
h
\end{array}\right)_{R} \sim\left(1,2, \frac{1}{6}\right), \\
& d_{R} \sim\left(1,1,-\frac{1}{3}\right), h_{L} \sim\left(1,1,-\frac{1}{3}\right), \\
& \left(\begin{array}{ll}
v & \psi_{2}^{+} \\
e & \psi_{2}^{0}
\end{array}\right)_{L} \sim(2,2,0),\left(\begin{array}{l}
S \\
e
\end{array}\right)_{R} \sim\left(1,2,-\frac{1}{2}\right), \\
& \left(\begin{array}{c}
\psi_{1}^{0} \\
\psi_{1}^{-}
\end{array}\right)_{L} \sim\left(2,1,-\frac{1}{2}\right) .
\end{aligned}
$$

The would-be $\bar{h}_{L} d_{R}$ term and the coupling of the two lepton fermion doublets through the $\eta$ scalar bidoublet are forbidden by an assumed $Z_{2}$ or $U(1)$ symmetry, the origin of which in the case of $[S U(3)]^{3}$ was discussed already [11]. This structure was recognized originally [13] to eliminate the presence of flavor changing neutral currents, but was subsequently used to accommodate dark matter in a simpler version where $(v, e)$ is an $S U(2)_{L}$ doublet without being part of an $S U(2)_{L} \times S U(2)_{R}$ 
bidoublet. In these dark left-right models (DLRM) [14, 15, 16], $S$ is a possible dark-matter candidate but its stability depends on an imposed global $U(1)$ symmetry. Recently, a model with an imposed gauge $U(1)$ symmetry has also been proposed [17].

The DLRM particle content is naturally embedded in an $[S U(3)]^{4}$ model [18] where leptonic color $S U(3)_{l}$ [19, 20] has been added. Under $S U(3)_{q} \times S U(3)_{L} \times S U(3)_{l} \times S U(3)_{R}$, the fermion chain is given by

$$
\begin{aligned}
& \left(\begin{array}{lll}
d & u & h \\
d & u & h \\
d & u & h
\end{array}\right),\left(\begin{array}{lll}
x_{1} & x_{2} & v \\
y_{1} & y_{2} & e \\
z_{1} & z_{2} & n
\end{array}\right),\left(\begin{array}{lll}
z_{1}^{c} & y_{1}^{c} & x_{1}^{c} \\
z_{2}^{c} & y_{2}^{c} & x_{2}^{c} \\
n^{c} & e^{c} & v^{c}
\end{array}\right), \\
& \left(\begin{array}{lll}
h^{c} & h^{c} & h^{c} \\
u^{c} & u^{c} & u^{c} \\
d^{c} & d^{c} & d^{c}
\end{array}\right),
\end{aligned}
$$

where the $S U(2)_{R}$ fermion doublet is now denoted $\left(e^{c}, n^{c}\right)$, with $n^{c}$ a dark-matter candidate, again after an imposed symmetry. Here $S U(3)_{l}$ has an unbroken $S U(2)_{l}$ subgroup which serves to confine the half-charged fermions $(x, y, z)$ in the same way that $S U(3)_{q}$ confines the quarks with one-third and twothird charges. Their phenomenology at a future $e^{-} e^{+}$collider has been discussed recently [21, 22]. It was also ascertained that the scalar singlet in an $\left(1,3,1,3^{*}\right)$ multiplet is a better darkmatter candidate.

There is another $[S U(3)]^{4}$ version [23] without leptonic color. The extra $S U(3)_{D}$ allows two fermion bidoublets, i.e. $S U(2)_{L} \times S U(2)_{D}$ and $S U(2)_{R} \times S U(2)_{D}$, which have the same form as $\psi$ with neutral components as dark matter. The former is ruled out because the neutral components couple to the $\mathrm{SM} \mathrm{Z}$ boson, but the latter could work. This idea has only been implemented recently in an explicit $S U(3)_{C} \times U(1)_{B-L} \times S U(2)_{L} \times$ $S U(2)_{R} \times S U(2)_{D}$ model [24]. It is a genuine first example of predestined $U(1)$ dark matter, in analogy to baryon and lepton numbers but unrelated to them.

The best known gauge $U(1)$ extension is just $S U(3)_{C} \times$ $S U(2)_{L} \times U(1)_{Y} \times U(1)_{B-L}$, under which

$$
\begin{aligned}
\left(\begin{array}{l}
u \\
d
\end{array}\right)_{L} & \sim\left(3,2, \frac{1}{6}, \frac{1}{3}\right), \\
u_{R} & \sim\left(3,1, \frac{2}{3}, \frac{1}{3}\right), d_{R} \sim\left(3,1,-\frac{1}{3}, \frac{1}{3}\right), \\
\left(\begin{array}{l}
v \\
e
\end{array}\right)_{L} & \sim\left(1,2,-\frac{1}{2},-1\right), \\
e_{R} & \sim(1,1,-1,-1), v_{R} \sim(1,1,0,-1) .
\end{aligned}
$$

Note that $v_{R}$ is required for $U(1)_{B-L}$ to be anomaly-free. To break $U(1)_{B-L}$, a neutral singlet $\zeta$ with 2 units of $B-L$ charge is the conventional choice, in which case $v_{R}$ gets a large Majorana mass, and the canonical seesaw mechanism allows $v_{L}$ to acquire a small Majorana mass. If $\zeta$ has 3 units of $B-L$ charge [25, 26, 27], then $v$ is a Dirac neutrino. In either case, $B$ is conserved. The one Higgs doublet $\Phi \sim(1,2,1 / 2,0)$ is the same as in the SM.

Consider now a fermion singlet or triplet which is also trivial under $U(1)_{B-L}$ :

$$
S \sim(1,1,0,0), \quad \Sigma=\left(\Sigma^{+}, \Sigma^{0}, \Sigma^{-}\right) \sim(1,3,0,0) .
$$

Neither would couple to $\Phi$ and a lepton doublet because of $U(1)_{B-L}$. Hence they are predestined dark matter. Again $S$ is completely decoupled, but $\Sigma$ works well as dark matter, as already mentioned in the case of left-right models without an $S U(2)_{L}$ scalar doublet.
As for scalar dark matter, assuming that $\zeta \sim(1,1,0, n)$ is used to break $U(1)_{B-L}$, then a scalar triplet

$$
\chi=\left(\chi^{+}, \chi^{0}, \chi^{-}\right) \sim\left(1,3,0, n^{\prime}\right)
$$

would have an $U(1)$ dark symmetry if $n^{\prime}$ is not zero, or $\pm n$. In that case, $\chi^{0}$ would couple to the $U(1)_{B-L}$ gauge boson as well as the SM Higgs boson, and be seriously constrained by present data. If a scalar singlet dark-matter candidate is desired, then it should be charged under $U(1)_{B-L}$, but not $\pm n$ or $\pm 2 n$ or $\pm 3 n$ [28].

Instead of $U(1)_{B-L}$ to support Majorana seesaw neutrino masses with heavy right-handed singlets $v_{R}$, a peculiar anomaly-free $U(1)_{X}$ may be used with heavy triplet fermions

$$
\Sigma_{R}=\left(\Sigma^{+}, \Sigma^{0}, \Sigma^{-}\right)_{R} \sim\left(1,3,0, n_{6}\right) .
$$

Together with

$$
\begin{aligned}
\left(\begin{array}{l}
u \\
d
\end{array}\right)_{L} & \sim\left(3,2, \frac{1}{6}, n_{1}\right), \\
u_{R} & \sim\left(3,1, \frac{2}{3}, n_{2}\right), d_{R} \sim\left(3,1,-\frac{1}{3}, n_{3}\right), \\
\left(\begin{array}{l}
v \\
e
\end{array}\right)_{L} & \sim\left(1,2,-\frac{1}{2}, n_{4}\right), \quad e_{R} \sim\left(1,1,-1, n_{5}\right),
\end{aligned}
$$

it was shown some years ago [29, 30, 31] and studied more recently [32, 33 that for $3 n_{1}+n_{4} \neq 0$, an anomaly-free solution exists per family if

$$
\begin{aligned}
& n_{2}=\frac{1}{4}\left(7 n_{1}-3 n_{4}\right), \quad n_{3}=\frac{1}{4}\left(n_{1}+3 n_{4}\right), \\
& n_{5}=\frac{1}{4}\left(-9 n_{1}+5 n_{4}\right), \quad n_{6}=\frac{1}{4}\left(3 n_{1}+n_{4}\right) .
\end{aligned}
$$

The scalar sector consists of a singlet $\left(1,1,0,\left(3 n_{1}+n_{4}\right) / 2\right)$ and two doublets $\left(1,2,1 / 2,3\left(n_{1}-n_{4}\right) / 4\right)$ and $\left(1,2,1 / 2,\left(9 n_{1}-\right.\right.$ $\left.\left.n_{4}\right) / 4\right)$. The singlet and triplet fermions of Eq. (19) are thus also predestined dark matter because neither would couple to either scalar doublet and a lepton doublet unless $3 n_{1}+n_{4}=0$. As for the scalar triplet of Eq. (20), an $U(1)$ dark symmetry would emerge if $n^{\prime}$ is not zero, or $\pm\left(3 n_{1}+n_{4}\right) / 2$, or $\pm\left(3 n_{1}+n_{4}\right)$. Again, $\chi^{0}$ would then couple to the $U(1)_{X}$ gauge boson as well as the two Higgs doublets, and be seriously constrained by present data.

In well-motivated gauge extensions of the SM, there are often simple fermion or scalar multiplets, i.e. singlets, bidoublets, and triplets, which may be stable without any imposed symmetry. Their neutral components are often automatically the lightest and thus predestined to be dark matter. In this paper, several examples are discussed in the context of left-right and $U(1)$ extensions of the SM. In the former, many examples are already known [5, 7, 8, 9] which involve mostly fermions. It is pointed out here that the absence of an $S U(2)_{L}$ scalar doublet [3] allows the $S U(2)_{L}$ scalar triplet $\chi_{L}$ of Eq. (8) to be dark matter as well. In the latter, whether $U(1)_{B-L}$ or $U(1)_{X}$ is used, there are also fermion and scalar triplets which could be predestined dark matter. The stabilizing dark symmetry is either $Z_{2}$ or $U(1)$.

\section{ACKNOWLEDGEMENTS}

This work was supported in part by the U. S. Department of Energy Grant No. DE-SC0008541. 


\section{References}

[1] M, Cirelli, N. Fornengo, and A. Strumia, Nucl. Phys. B753, 178 (2006).

[2] E. Ma, Phys. Rev. D69, 011301(R) (2004).

[3] A. Aranda, J. L. Diaz-Cruz, E. Ma, R. Noriega, and J. Wudka, Phys. Rev. D80, 115003 (2009).

[4] M. Sher, Phys. Rev. D52, 3136 (1995).

[5] C. Garcia-Cely and J. Heeck, JCAP 1603, 021 (2016).

[6] E. Ma and D. Suematsu, Mod. Phys. Lett. A24, 583 (2009).

[7] J. Heeck and S. Patra, Phys. Rev. Lett. 115, 121804 (2015).

[8] C. Arbelaez, M. Hirsch, and D. Restrepo, Phys. Rev. D95, 095034 (2017).

[9] S. K. Agarwalla, K. Ghosh, and A. Patra, arXiv:1803.01670 [hep-ph].

[10] E. Ma, Phys. Rev. Lett. 115, 011801 (2015).

[11] C. Kownacki, E. Ma, N. Pollard, O. Popov, and M. Zakeri, Phys. Lett. B777, 121 (2018).

[12] P. V. Dong, D. T. Huong, F. Queiroz, J. W. F. Valle, and C. A. Vaquera-Araujo, arXiv:1710.06951.

[13] E. Ma, Phys. Rev. D36, 274 (1987).

[14] S. Khalil, H.-S. Lee, and E. Ma, Phys. Rev. D79, 041701(R) (2009).

[15] S. Khalil, H.-S. Lee, and E. Ma, Phys. Rev. D81, 051702(R) (2010).

[16] S. Bhattacharya, E. Ma, and D. Wegman, Eur. Phys. J. C74, 2902 (2014).

[17] C. Kownacki, E. Ma, N. Pollard, O. Popov, and M. Zakeri, Eur. Phys. J. C78, 148 (2018).
[18] K. S. Babu, E. Ma, and S. Willenbrock, Phys. Rev. D69, 051301(R) (2004).

[19] R. Foot and H. Lew, Phys. Rev. D41, 3502 (1990).

[20] R. Foot, H. Lew, and R. R. Volkas, Phys. Rev. D44, 1531 (1991).

[21] C. Kownacki, E. Ma, N. Pollard, O. Popov, and M. Zakeri, Phys. Lett. B769, 267 (2017).

[22] C. Kownacki, E. Ma, N. Pollard, O. Popov, and M. Zakeri, Nucl. Phys. B928, 520 (2018).

[23] E. Ma, Mod. Phys. Lett. A20, 1953 (2005).

[24] E. Ma, arXiv:1712.08994 [hep-ph].

[25] E. Ma, I. Picek, and B. Radovcic, Phys. Lett. B726, 744 (2013).

[26] E. Ma and R. Srivastava, Phys. Lett. B741, 217 (2015).

[27] E. Ma, N. Pollard, R. Srivastava, and M. Zakeri, Phys. Lett. B750, 135 (2015).

[28] W. Rodejohann and C. E. Yaguna, JCAP 1512, 032 (2015).

[29] E. Ma, Mod. Phys. Lett. A17, 535 (2002).

[30] E. Ma and D. P. Roy, Nucl. Phys. B644, 290 (2002).

[31] S. M. Barr and I. Dorsner, Phys. Rev. D72, 015011 (2005); See also S. M. Barr, B. Bednarz, and C. Benesh, Phys. Rev. D34, 235 (1986).

[32] R. Adhikari, J. Erler, and E. Ma, Phys. Lett. B672, 136 (2009).

[33] R. Adhikari, D. Borah, and E. Ma, Phys. Lett. B755, 414 (2016). 